\title{
Molecular Characterization of the Recombinant A-chain of a Type II Ribosome-Inactivating Protein (RIP) from Viscum album coloratum and Structural Basis on its Ribosome-Inactivating Activity and the Sugar-binding Properties of the B-chain
}

\author{
Wenhui Ye ${ }^{1}$, Ravi Prakash Reddy Nanga ${ }^{1}$, Cong Bao Kang ${ }^{1}$, Joo-Hye Song ${ }^{2}$, \\ Seong Kyu Song ${ }^{2}$ and Ho Sup Yoon ${ }^{1, *}$ \\ ${ }^{1}$ Division of Structural and Computational Biology, School of Biological Sciences, Nanyang Technological University, \\ 60 Nanyang Drive Singapore 637511, Singapore \\ ${ }^{2}$ Institute for Biomedical Research, Handong University, Buk-ku, Pohang, 791-940, South Korea
}

Received 20 February 2006, Accepted 19 May 2006

\begin{abstract}
Mistletoe (Viscum album) lectins, which are classified as a type II ribosome-inactivating protein (RIP) due to their unique biological function and the potential medical and therapeutic application in cancer cells, receive a rising attention. The heterodimeric glycoproteins contain the Achain with catalytic activity and the B-chain with sugar binding properties. In recent years, studies involving the lectins from the white berry European mistletoe (Viscum album) and the yellow berry Korean mistletoe (Viscum album coloratum) have been described. However, the detailed mechanism in exerting unique cytotoxic effect on cancer cells still remains unclear. Here, we aim to understand and define the molecular basis and biological effects of the type II RIPs, through the studies of the recombinant Korean mistletoe lectin. To this end, we expressed, purified the recombinant Korean mistletoe lectin (rKML), and investigated its molecular characteristics in vitro, its cytotoxicity and ability to induce apoptotic cell death in cancer cells. To gain structural basis for its catalytic activity and sugar binding properties, we performed homology modeling studies based on the high degree of sequence identity and conserved secondary structure prediction between Korean and European, Himalayan mistletoe lectins, and Ricin.
\end{abstract}

Keywords: Lectin, Ribosome-inactivating protein, Sugar binding, Viscum album coloratum

\footnotetext{
* To whom correspondence should be addressed.

Tel: 65 6316-2846; Fax: 65 6791-3856

E-mail: hsyoon@ntu.edu.sg
}

\section{Introduction}

Mistletoe (Viscum album) is a semi-parasitic plant that lives on different types of trees including the oak. The lectins purified from the mistletoe extracts have been shown to have anti-cancer activities and immunomodulatory effects (Hajto et al., 1990; Schumacher et al., 2000). The lectins are heterodimeric glycoproteins containing the A-chain with cytotoxic activity and the B-chain with sugar binding properties, and are classified as type II ribosome-inactivating proteins (RIP) due to its RNA N-glycosidase activity (Endo et al., 1988). Previously, it was shown that the structure of the European mistletoe lectin-I, a type II RIP, shares a high degree of structural similarities with ricin, a plant toxin, showing that the A-chain comprises three distinct individual domains with the active site at the interface formed by the three domains (Krauspenhaar et al., 1999). The B-chain contains two domains with similar configuration. The A-and B-chains are linked by a disulfide bond. So far, more than 15 type II RIPs have been identified, and they appear to be very homologous (Montfort et al., 1987; Peumans et al., 2001). Recently, the cDNAs for European and Korean Mistletoe lectins, which belong to type II RIP, have been cloned, indicating that the Achains of Korean mistletoe lectins (KML) show more than 90 $\%$ of identity to that of European mistletoe lectin-1 (EML-1) at primary structure level, while its B-chains show $83 \%$ sequence identity with that of European one (Eck et al., 1999; Park et al., 2001). Korean mistletoe lectins show the existence of several isotypes (Park et al., 2001). Interestingly, different sugar binding specificity was also suggested; some bind to Dgalactose or some bind to N-acetyl-D-galactosamine or both. It is speculated that the difference in the sugar binding specificity of the B-chains may play an important role in determining the selective cytotoxicity of the lectins with 
cancer cells by interacting with a putative receptor on the cell surface. The cellular cytotoxicity of the lectins appears to require both the $\mathrm{A}$ - and $\mathrm{B}$-chains. So, recognition and internalization of the B-chain of the RIP via a putative receptor might be critical and prerequisite for the cytotoxic activity of the A-chain.

Over the last few years, several type II RIPs including the lectins from the white berry European mistletoe (Viscum album) and the yellow berry Korean mistletoe (Viscum album coloratum) have been described for a potential medicinal application (Yoon et al., 1995; Schumacher et al., 2000) However, the detailed molecular mechanism in exerting cytotoxic effect on cancer cells still remains to be unclear. Here, we aim to understand and define the molecular basis and biological effects of the type II RIPs, through the studies of the recombinant Korean mistletoe lectin. Towards this end, we expressed, purified the recombinant Korean mistletoe lectin (rKML), and investigated its molecular characteristics in vitro, its cytotoxicity and ability to induce apoptotic cell death in cancer cells, and then to gain structural basis for its ribosome inactivating activity and sugar binding properties, we performed homology modeling studies based on the high degree of sequence identity and conserved secondary structure prediction between Korean and other mistletoe lectins including European, Himalayan, and Ricin.

\section{Materials and Methods}

Materials. $\mathrm{Ni}^{2+}$-NTA resin was from Qiagen. Protein molecular weight markers were from Bio-Rad Laboratories. HiPrep 16/60 Sephacryl S-200 was from Amersham Biosciences. Escherichia coli BL21 (DE3) and carbenicillin were from Invitrogen. pET plasmids were from Novagen. Isoporpyl-thio- $\beta$-D-galactopyranoside (IPTG) was from Promega. Dulbecco's modified Eagle's medium (DMEM) was from Invitrogen. BioPORTER was from Gene Therapy Systems. Complete-mini protease inhibitors tablets were from Roche Applied Science. Generic chemicals were purchased from Sigma-Aldrich.

Cell cultures. MCF-7/caspase-3(+) cell line was kindly provided by Dr. Alan Porter (Institute of Molecular and Cell Biology). Cells were cultured at $37^{\circ} \mathrm{C}$ in a humidified $\mathrm{CO}_{2}$ incubator under DMEM supplemented with $10 \%$ fetal bovine serum, $2 \mathrm{mM}$ L-glutamine, $100 \mathrm{units} / \mathrm{ml}$ penicillin and $100 \mu \mathrm{g} / \mathrm{ml}$ streptomycin.

Expression and purification of recombinant Korean mistletoe lectin. The construction of expression plasmids containing the cDNAs coding for the Korean mistletoe lectins was previously described (Park et al., 2001). The plasmids containing rKML-1A (pETKML-1A) and rKML-1B (pETKML-1B) were transformed into $E$. coli BL21 (DE3) cells. The proteins were induced by adding IPTG to $1 \mathrm{mM}$ when $\mathrm{Abs}_{600}$ was $0.8-1.0$. After induction at $30^{\circ} \mathrm{C}$ for $2 \mathrm{~h}$, the cells were harvested by centrifugation at $8,000 \times \mathrm{g}$ for $10 \mathrm{~min}$. The cells were suspended in a resuspension buffer $(20 \mathrm{mM}$ phosphate, $\mathrm{pH} 7.8,0.5 \mathrm{M} \mathrm{NaCl}, 5 \mathrm{mM}$ 2-mercaptoethanol) and then disrupted by ultrasonication for $10 \mathrm{~min}$ on ice. The cell lysate was centrifuged at $20,000 \times g$ for $25 \mathrm{~min}$. The clear supernatant was loaded to $\mathrm{Ni}^{2+}$-NTA column, and the column was washed with 30 column volumes of a washing buffer $(20 \mathrm{mM}$ phosphate, $\mathrm{pH}$ 7.2, $1 \mathrm{M} \mathrm{NaCl}, 20 \mathrm{mM}$ imidazole, $5 \mathrm{mM}$ 2-mercaptoethanol) and eluted with an elution buffer $(20 \mathrm{mM}$ phosphate, $\mathrm{pH} 6.0,0.5 \mathrm{M}$ $\mathrm{NaCl}, 5 \mathrm{mM}$ 2-mercaptoethanol, $0.5 \mathrm{M}$ imidazole). After collecting the eluted fractions from the $\mathrm{Ni}^{2+}$-NTA affinity column, the protein samples were loaded at $1 \mathrm{ml} / \mathrm{min}$ on to Sephacryl S-200 gel filtration column in $50 \mathrm{mM}$ Tris-Cl, $\mathrm{pH} 8.0,150 \mathrm{mM} \mathrm{NaCl}, 1 \mathrm{mM}$ dithiothreitol (DTT), $0.01 \%$ sodium azide. The purified protein samples were analyzed by $12.5 \%$ sodium dodecyl sulfate-polyacrylamide gel electrophoresis (SDS-PAGE). The concentration of purified proteins was determined by Bio-Rad Laboratories protein assay kit.

Circular Dichroism (CD) spectroscopy. The protein in $1 \times$ phosphate buffered saline (PBS) was concentrated to $1 \mathrm{mg} / \mathrm{ml}$ and $\mathrm{CD}$ was measured at $298 \mathrm{~K}$ in the range of $190-250 \mathrm{~nm}$ using a Chirascan spectropolarimeter (Applied Photophysics Limited, UK) in continuous scanning mode. The acquisition parameters for the CD spectrum were $20 \mathrm{~nm} / \mathrm{min}$ with a $4 \mathrm{~s}$ response and a $2-\mathrm{nm}$ bandwidth. For the consistency of the experiment, the protein concentration was also determined at the end of the run.

NMR spectroscopy. The purified rKML-1A was concentrated to $0.5 \mathrm{mM}$ in a buffer containing $20 \mathrm{mM}$ phosphate, $\mathrm{pH} 7.0,20 \mathrm{mM}$ $\mathrm{NaCl}, 1 \mathrm{mM}$ DTT, $0.01 \% \mathrm{NaN}_{3}$, and $10 \% \mathrm{D}_{2} \mathrm{O}$. The NMR measurement was performed with water presaturation at $298 \mathrm{~K}$ on an Avance $700 \mathrm{MHz}$ spectrometer (Bruker) equipped with four-radio frequency channels and a triple resonance probe. The spectra were processed and analyzed by using Topspin (Bruker).

Assay of ribosome-inactivation activity of RIP. A nonradioactive assay for RIP activity was performed as described (Langer et al., 1996) by utilizing a coupled transcription/translation system. Briefly, in this highly sensitive assay the inhibition of protein synthesis by the rRNA-N-glycosidase activity RIPs was determined quantitatively by measuring luciferase activity in a cell-free rabbit reticulocyte lysate system (Promega).

Cell proliferation assay. Cells were seeded into 96-well plates at $5 \times 10^{3}$ cells per well. On the following day rKML-1A with the help of BioPORTER or KML was delivered into cells at different concentration. After $24 \mathrm{~h}, 20 \mu \mathrm{l}$ of combined MTS/PMS solution (Promega) was added to each well. After incubating the plate for $2 \mathrm{~h}$ at $37^{\circ} \mathrm{C}$ in a humidified, $5 \% \mathrm{CO}_{2}$ atmosphere, the absorbance of each well was measured at $490 \mathrm{~nm}$ using a microplate reader from Bio-Rad Labs.

Annexin-V-FLUOS staining. Approximately $2 \times 10^{5}$ cells/well were seeded in a 6-well plate. On the following day, cells were treated with rKM-A1/BioPORTER. After $24 \mathrm{~h}$, cells were harvested by trypsinization, and then resuspended in $100 \mu \mathrm{l}$ of Annexin-V-FLUOS staining solution (containing annexin Vfluorescein and propidium iodide) and incubated for $15 \mathrm{~min}$ at $15-$ $25^{\circ} \mathrm{C}$. The samples were analyzed with a fluorescence-activated cell sorter (FACS) (BD Biosciences) using $488 \mathrm{~nm}$ excitation and a $515 \mathrm{~nm}$ band pass filter for fluorescein detection, and a filter $>600 \mathrm{~nm}$ for propidium iodide detection. 
Homology modeling. Modeller 8v1 (Sali et al., 1993) was used to build the 3D structure of KML by taking the structure of European mistletoe (Viscum album) Lectin-I complexed with galactose (PDB code 1OQL) as template, and the model thus obtained was viewed with the help of InsightII interface. Finally, the KML model was checked for the stereochemical parameters by the program PROCHECK (Laskowski et al., 1993).

\section{Results}

Expression and purification of the recombinant Korean mistletoe lectin. Previously, the Korean mistletoe lectins were shown to exist in several isoforms (Park et al., 2001). In the present work, we chose KML-1A, which is one of the Achain isoforms, and KML-1B, which is one of the B-chain isoforms, for investigation. Efforts were made to obtain the recombinant Korean mistletoe lectin (rKML). Both the A- and the B-chains of rKML (rKML-1A and rKML-1B) were expressed separately in a bacterial expression system. Our results showed that the rKML-1A was expressed in a soluble form (Fig. 1A) while the rKML-1B was in an insoluble form. Several attempts, which included protocols using refolding and different expression conditions, were made to obtain the soluble form of the B-chain but failed to improve its solubility (data not shown). Here, therefore, we focused on the purification and molecular characterization of rKML-1A. After $\mathrm{Ni}^{2+}$-NTA affinity and Sephacryl S-200 gel filtration column chromatography, we were able to obtain more than $95 \%$ pure rKML-1A (Fig. 1A, lane 6). The protein was stable for several months when stored at $4^{\circ} \mathrm{C}$.

\section{Molecular characterization of the recombinant Korean mistletoe lectin A-chain (rKML-1A). To evaluate and study the characteristics of the purified rKML-1A, we first performed CD analysis (Fig. 1B), showing that the rKML-1A contains both $\alpha$-helix and $\beta$-sheet, and also suggests apparently folded pattern of a globular protein. To further probe whether the rKML-1A is correctly folded, we then monitored $1 \mathrm{D}{ }^{1} \mathrm{H}$ NMR spectra of rKML-1A (Fig. 1C). NMR is a good tool to define the correct folding of proteins. The 1D ${ }^{1} \mathrm{H}$ NMR spectrum of the rKML-1A shows the characteristics of properly folded proteins featuring good dispersion of resonance lines in the three spectral regions where the methyl protons, $\alpha$-protons, and amide protons are present and separated (Page et al., 2005). Taken together, our results indicate that the purified rKML-1A is correctly folded and ready for biochemical and structural studies.}

Ribosome-inactivating activity of the rKML-1A. To analyze the catalytic activity of the rKML-1A, the ribosomeinactivating activity of the rKML-1A was carried out using a coupled transcription/translation assay (Langer et al., 1996). A concentration range from 10 to $1000 \mu \mathrm{g} / \mathrm{ml}$ of the rKML$1 \mathrm{~A}$ was used to determine $\mathrm{IC}_{50}$. Our data showed that the in vitro synthesis of luciferase in a rabbit reticulocyte lysate system was inhibited by the addition of the purified rKML1A. To determine $\mathrm{IC}_{50}$ of the $\mathrm{rKML}-1 \mathrm{~A}$ and the natural KML, we separately measured the luciferase activity in the absence of the lectins and then normalized to $100 \%$ translational activity. As shown in Fig. 2, the calculated $\mathrm{IC}_{50}$ values for the rKML-1A and the native KML were $3.05 \mathrm{nM}$ and $3.23 \mathrm{nM}$, respectively. Our results here suggest that both the rKML-1A and the native KML carry similar levels of ribosome-inactivating activity under our assay condition used in this study.

The rKML-1A induces apoptosis in cancer cells. The natural Korean mistletoe lectin was shown to kill cancer cell by inducing apoptosis through modulating several molecules including caspases and telomerase (Lyu et al., 2002; Lyu et al., 2001; Yoon et al., 1999). In this study, to confirm and demonstrate the biological effects of the rKML-1A in cancer cells, we first performed cell proliferation assays in MCF7/ caspases-3 $(+)$ cells. The cytotoxic effects of the mistletoe lectins require both the A- and B-chains; the A-chain for catalytic activity and the B-chain for internalization (Peumans et al., 2001). Since the recombinant B-chain was not available, in this study, we employed a protein delivery reagent BioPORTER for the delivery of rKML-1A into cells, and then evaluated its cytotoxicity. The cytotoxicity of rKML1A was compared with that of the natural KML. We demonstrated that the growth of MCF7/caspases-3 (+) cells was inhibited by the treatment of rKML-1A and KML in a dose-dependent manner (Fig. 3). The cells apparently showed reduced cell viability at $1 \mu \mathrm{g} / \mathrm{ml}$ of rKML-1A, whereas the natural KML started showing its cytotoxic effect at $10 \mathrm{ng} / \mathrm{ml}$ level. These data suggest that the efficiency using BioPORTER as an artificial delivery vehicle for transporting rKML-1A into cells is considerably lower compared to that of the natural KML. To further define whether the growth inhibition of cancer cells by rKML-1A is caused by the induction of apoptosis, the caspases-3 activity measurement and annexinV-FLUOS staining were performed after the cells were treated with the rKML-1A/BioPORTER (Fig. 4). Our results showed that significant increase of caspase- 3 activity was observed as a result of rKML-1A treatment compared to the control cells without protein; the caspase-3 activity increased 1.93 fold at $1 \mu \mathrm{g} / \mathrm{ml}$ of rKML-1A and 3.03 fold at $10 \mu \mathrm{g} / \mathrm{ml}$ of rKML-1A (Fig. 4A). Our FACS data also demonstrated that the degree of apoptotic cells was $10.74 \%$ at $1 \mu \mathrm{g} / \mathrm{ml}$ of rKML-1A and increased to $21.16 \%$ at $10 \mu \mathrm{g} / \mathrm{ml}$ of rKML-1A, respectively (Fig. 4B), indicating a significant increase in apoptotic cell population over control cells which show marginal apoptosis to $3.48 \%$. Taken together, our data suggest that the cytotoxic effect of rKML-1A in the cancer cells appears to be due to the induction of apoptosis.

Homology modeling of Korean mistletoe lectin (KML). Sequence comparison of KML with that of European mistletoe lectin-1 shows a high degree of homology (Eck et al., 1999; Park et al., 2001) KML-1A chain shows 92.9\% 

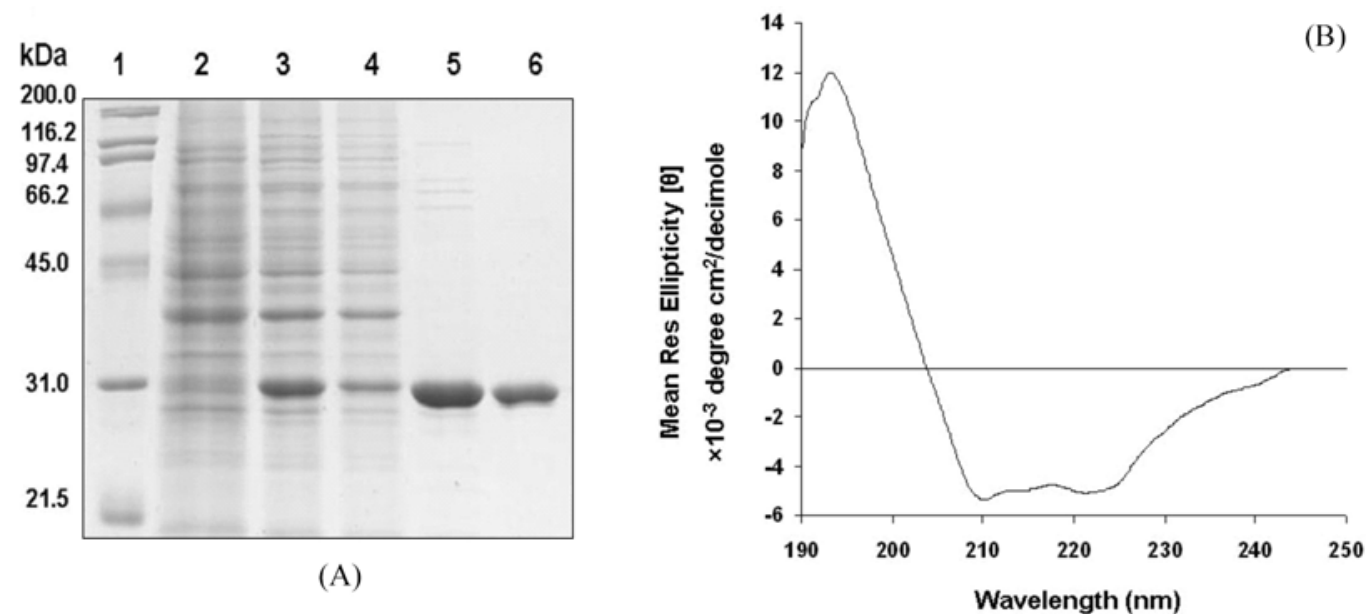

(A)

(C)

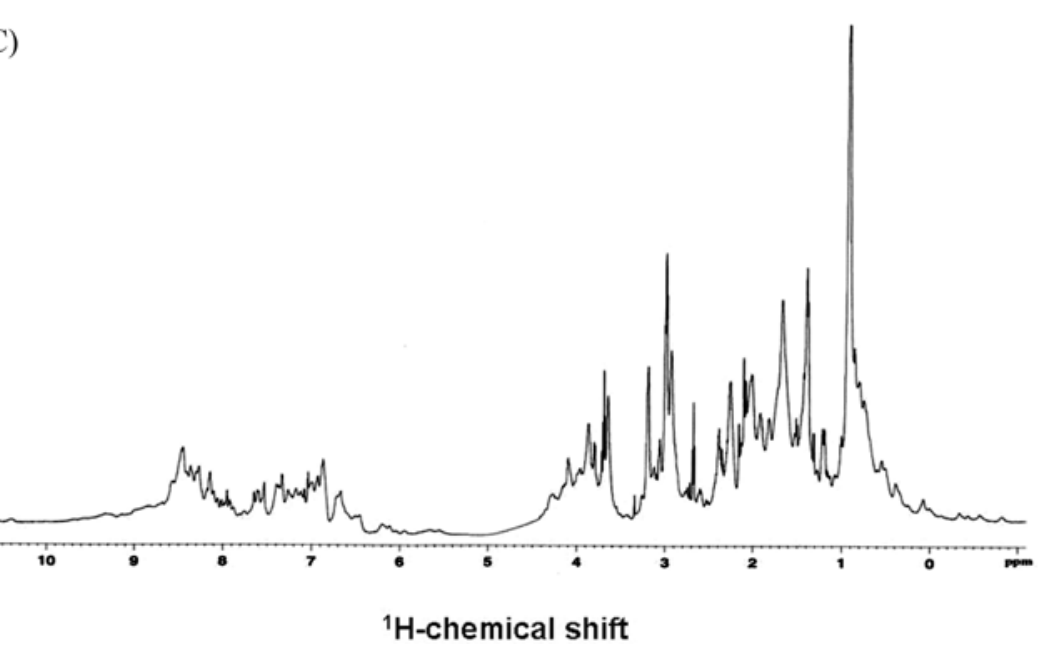

Fig. 1. Expression, purification and characterization of the rKML-1A (A) the rKML1-A1 was expressed in E. coli BL21 (DE3) cells, purified by $\mathrm{Ni}^{2+}$-NTA affinity resin and further purified by Sephacryl S-200 gel filtration chromatography. Protein samples at various stages were analyzed by $12 \%$ SDS-PAGE 1: lane1, MW marker; lane 2,3, before and after IPTG induction; lane 4, the supernatant of the induced sample; lane 5, elution from $\mathrm{Ni}^{2+}$-NTA affinity resin, lane 6, the rKML-1A after Sephacryl S-200. (B) Circular Dichroism (CD) analysis of the rKML-1A. The purified rKML-1A in PBS buffer was concentrated to $1 \mathrm{mg} / \mathrm{ml}$. The spectrum was recorded in the range of the wavelengths from $190 \mathrm{~nm}$ to $250 \mathrm{~nm}$. (C) $1 \mathrm{D}{ }^{1} \mathrm{H}$ NMR analysis of the rKML-1A. The rKML-1A was purified and concentrated to $0.5 \mathrm{mM}$ in the buffer containing $20 \mathrm{mM}$ phosphate, $\mathrm{pH} 7.0,20 \mathrm{mM} \mathrm{NaCl}, 1 \mathrm{mM} \mathrm{DTT}, 0.01 \% \mathrm{NaN}_{3}$, and $10 \% \mathrm{D}_{2} \mathrm{O}$ was used. The $1 \mathrm{D}{ }^{1} \mathrm{H}$ spectrum was recorded on Avance 700MHz NMR spectrometer (Bruker) as described in "Materials and methods".

sequence identity and less conserved B-chain, KML-1B, shows $83.2 \%$ sequence identity (Fig. 5). This high degree sequence homology led us to investigate the 3-dimensional model of KML. The KML model obtained was checked by the program PROCHECK, yielding $93 \%$ of the backbone conformations within the most favored regions of the Ramachandran plot. For the X-ray structure of 1OQL (Niwa et al., 2003), the percent of the backbone conformations within the most favored regions of the Ramachandran plot are $87.6 \%$. We have also taken the X-ray structures of Himalayan mistletoe lectin (PDB ID:1YF8) \& Ricin (PDB ID:2AAI) for analyzing the root mean square deviation (RMSD) of the $\mathrm{C}^{\alpha}$ trace. When analyzing the structural alignment of the $\mathrm{C}^{\alpha}$ traces between KML and the EML-1, HML and Ricin, the
RMSD between KML and the EML-1, HML and Ricin was $0.218,0.533$ and $0.787 \AA$, respectively which is shown in Fig 6A. This suggests that the homology model we obtained is reliable. The structure generated from homology modeling in our studies is highly homologous to that of European ML-1, indicating that the overall three-dimensional topologies between two proteins are nearly identical (Fig. 6B).

The catalytic site of KML is conserved. As with the A-chain of EML-1, the A-chain of KML-1 is supposed to excise a specific adenine base from a highly conserved loop region of $28 \mathrm{~S}$ rRNA (Endo et al., 1998). Our data show that the location of the active site region of the KML is in a prominent cleft in the structure shown (Fig 6C). The alignment of the 


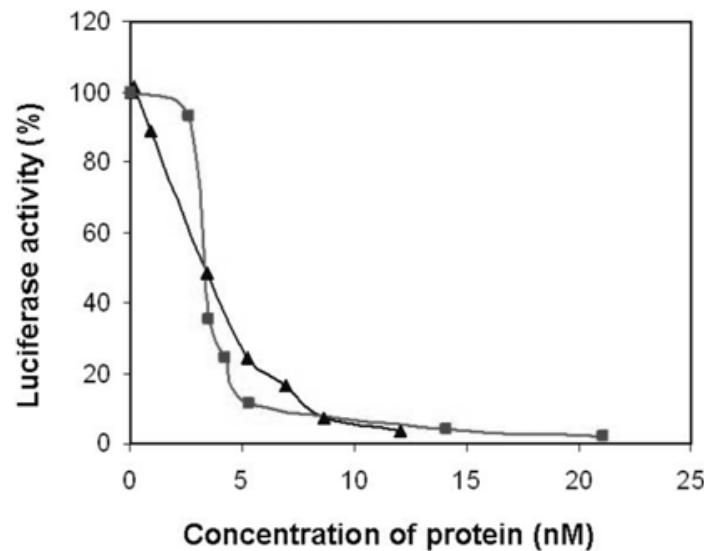

Fig. 2. Ribosome-inactivating activity of the rKM-1A and the KML. Ribosome-inactivating activity of the rKML-1A ( $\boldsymbol{\square})$ and the KML $(\boldsymbol{\Delta})$ was determined by measuring the degree of inhibition of luciferase translation in a rabbit reticulocyte lysate system. The luciferase activity of sample (without protein) was normalized to $100 \%$ translational activity. And the data from samples with increasing concentration of rKML-1A or KML were corrected accordingly. $\mathrm{IC}_{50}$ was about $3.05 \mathrm{nM}$ for rKML$1 \mathrm{~A}$ and $3.23 \mathrm{nM}$ for KML calculated by a three-parameter-fit equation.

amino acid sequences indicates that all four invariant residues in the active site of the KML-1A are conserved throughout the wide range of RIPs (Katzin et al., 1991) and the overall architecture of the active site cleft region remains almost constant as well. As seen in Fig. 7A, the key catalytic residues Tyr76, Tyr115, Glu165, and Arg168 of the A-chain of EML-1 (Niwa et al., 2003), which are conserved, are superimposed with KML-1A, showing a high degree of structural similarity with only a minor variation. One N-linked glycosylation site is present in the EML-1, and the residue involved is Asn112. On the other hand, in the KML-1A, Asn112 is replaced by Thr112. Whether this has any influence on the overall antitumor activity of KML-1A needs to be explored.
Molecular basis of glycosylation and sugar binding characteristics of KML. As with the EML-1B, the KML-1B is supposed to help in the transport of the A-chain into cells by first binding to specific sugar residues on the cell surface and subsequently through endocytosis (Narayanan et al., 2005). From the sequence comparison, it is clear that out of the three $\mathrm{N}$-glycosylation sites in the EML-1B, only one N-glycosylation site is conserved in the KML-1B. The three N-glycosylation sites (N-X-S/T motif) present in EML-1B are NGS (61-63), NGT (96-98), NDT (136-139) and the corresponding residues at the same sites in the KML-1B are NGR (61-63), NGI (9698) and NDT (139-141). In the KML-1B, the residues mutated at $1^{\text {st }}$ and $2^{\text {nd }} \mathrm{N}$-glycosylation site that leads to the loss of glycosylation are R63 and I98, respectively (Fig. 5).

Sugar binding characteristics of KML was also studied by molecular modeling. The hydrogen bonding between- galactose and EML-1B was determined after adding the hydrogens to the protein at $\mathrm{pH} 7$. The residues of the EML-1B involved in the hydrogen bonding with galactose at the N-terminal site are Asp23, Asp26, Lys41 and Asn47 with Trp38 providing the hydrophobic contact, while at the C-terminal site the residues involved are Asp235, Gln238 and Asn256 with Tyr249 providing the hydrophobic contact; In EML-1B, both the Asp23 \& Asp235 satisfies the donor-acceptor distance criteria of $3.9 \AA$ for the hydrogen bonding. But when we added the hydrogens to the protein at $\mathrm{pH} \mathrm{7,} \mathrm{the} \mathrm{hydrogen-acceptor} \mathrm{distance} \mathrm{criteria}$ of $2.5 \AA$ was not met, due to which we were unable to see the hydrogen bonding in our model. But the corresponding residues in KML-1B are Asp23 \& Asp238 are very well conserved. We then superimposed the KML structure with that of EML and then studied the differences at the both Cand N-terminal sites. At the N-terminal site while Asp23, Lys41, Asn47 and Trp38 are conserved, the only residue mutated in KML-1B was Glu26 instead of Asp26 (Fig. 7B). At the C-terminal site while Asp238, Asn259 are conserved, the residue mutated was Glu241 instead of Gln238 and the Tyr249 which provides the hydrophobic contact is mutated to Phe252 in KML-1B (Fig. 7D). Since only the main chain

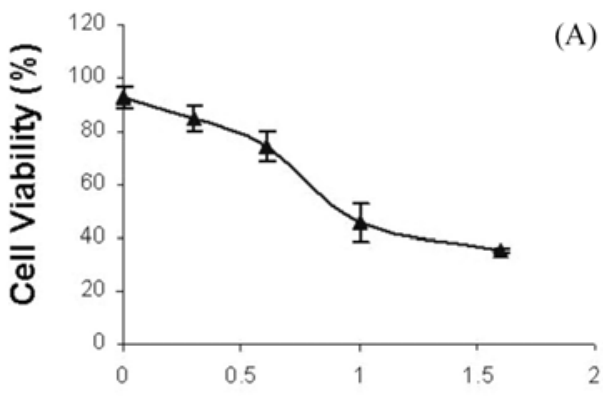

Log concentration of $\mathrm{KML}(\mathrm{ng} / \mathrm{ml})$

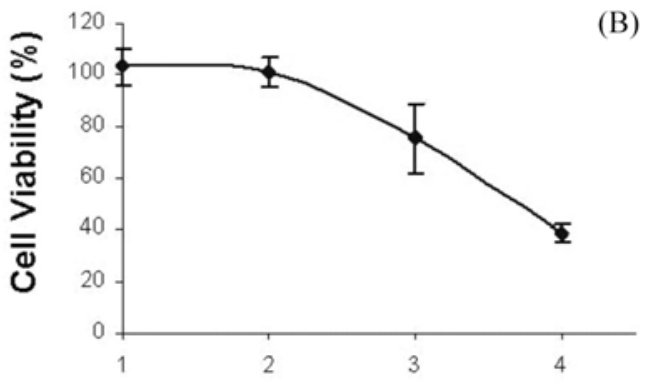

Log concentration of $\mathrm{rKML}-1 \mathrm{~A}(\mathrm{ng} / \mathrm{ml})$

Fig. 3. Cytotoxicity of the rKM-A1 and the KML on MCF-7/caspase-3 positive Cells. Cells were treated with the KML (A: $\mathbf{\Delta}$ ) and the rKML-1A (B: $\mathbf{a})$ for $24 \mathrm{~h}$. Cell viability was measured by MTS assay at $490 \mathrm{~nm}$. Cell viability was expressed as a percent relative to untreated control. Experiment was done once in triplicate. Data was presented as the mean \pm SD $(n=3)$. 


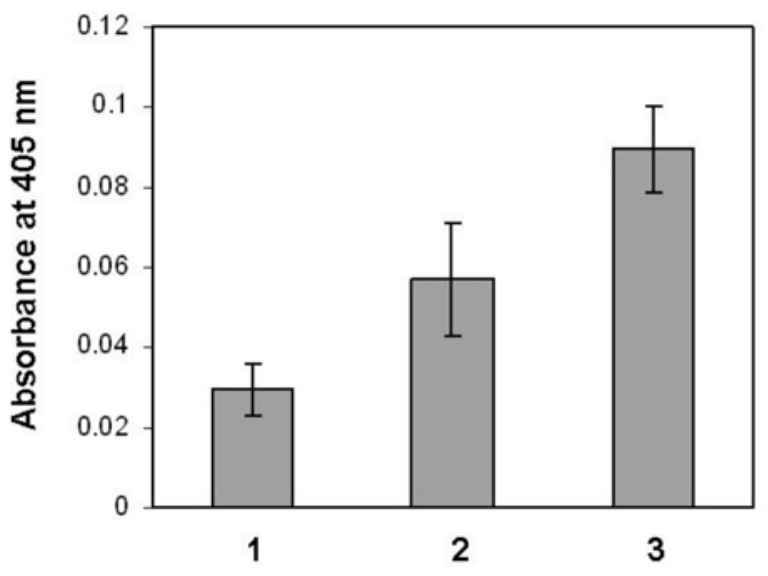

(A)

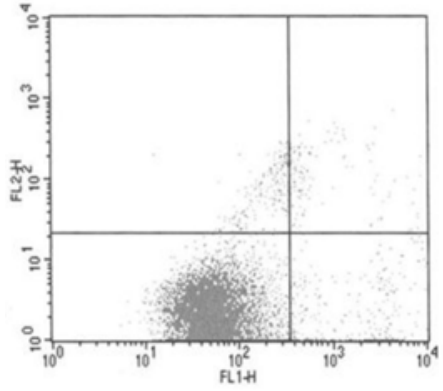

1

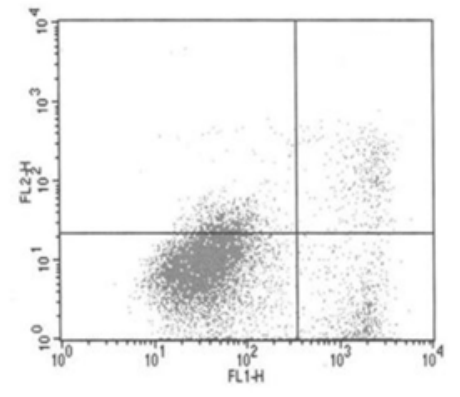

2

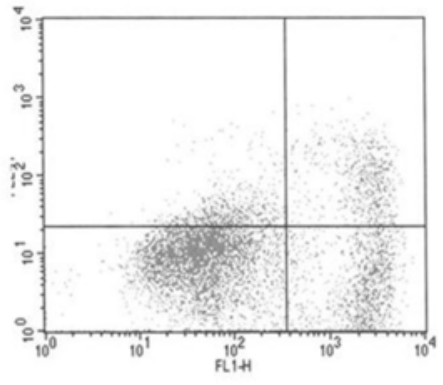

3

(B)

Fig. 4. rKML-1A induces apoptosis. (A) Cells were incubated in the absence or presence of rKML-1A. At $24 \mathrm{~h}$ after the treatment, caspase-3 activity was analyzed. 1, control cells without protein; 2, cells treated with $1 \mu \mathrm{g} / \mathrm{ml}$ the $\mathrm{rKML}-1 \mathrm{~A} ; 3$, cells treated with $10 \mu \mathrm{g} /$ $\mathrm{ml} \mathrm{ml} \mathrm{rKML-1A.} \mathrm{Data} \mathrm{was} \mathrm{presented} \mathrm{as} \mathrm{the} \mathrm{mean} \pm \mathrm{SD}(\mathrm{n}=3)$. (B) MCF-7/caspase-3 (+) cells were treated with different concentration of the rKML-A1 by BioPORTER reagent. 1, control cells without the rKML-1A; 2, cells treated with $1 \mu \mathrm{g} / \mathrm{ml} \mathrm{rKML}-1 \mathrm{~A}$; 3 , cells treated with $10 \mu \mathrm{g} / \mathrm{ml}$ the rKML-1A. 24 hours after the treatment, cells were stained with Annexin-V-Fluorescein and Proidium iodide, and analyzed by FACS. The apoptotic cells were calculated by measuring the lower right area.

atoms of Asp26 and Gln 238 in EML-1B are involved in the hydrogen bonding, the corresponding residues mutated into Glu26 and Glu241 in KML-1B are speculated to make little influence on the galactose binding. Also the Tyr249 in EML1B mutated to Phe252 in KML-1B can still provide the hydrophobic contact for galactose. From the above studies it is clear that the catalytic and galactose binding sites of KML are similar and pretty much conserved as that of EML-1. Therefore, KML might have a bit different degree of biological effect or activity compared to that of EML-1.

Recently, a third sugar binding site was reported for Himalayan mistletoe lectin (HML) (Mishra et al., 2005). We then performed multiple sequence alignment of KML, EML1, HML and Ricin using clustalW program (Thompson et al., 1994) which is shown in Fig. 5. From the sequence alignment, interestingly all the residues in EML at the third sugar binding site are absolutely conserved while the residues mutated in KML are Gly85, Tyr79 and the residues mutated in Ricin are Tyr78, Ala84. We then superimposed KML structure with Xray structure of HML after adding the hydrogens at $\mathrm{pH} 7$ and then studied the differences at the third sugar binding site which is shown in Fig.7C. The residues of HML-B involved in providing hydrophobicity to galactose \& glucose moiety of lactose are Tyr66, Phe75 \& Tyr64 and the corresponding residues in KML-1B are Tyr70, Tyr79 \& Tyr68. The residues of HML-B involved in hydrogen bonding with galactose and glucose moiety of lactose are Thr63, Val81 \& Tyr71 and the corresponding residues in KML-1B are Thr67, Gly85 \& Tyr75. Since only main chain amide nitrogen of Val81 in HML-B is involved in the hydrogen bonding, the corresponding residue mutated into Gly85 is speculated to make little influence on the binding of lactose. Also the Phe 75 in HML-B mutated to Tyr79 in KML-1B can still provide the hydrophobic contact for galactose moeity of lactose; In HML-B, both the Thr63 \& Tyr71 satisfies the donor-acceptor distance criteria of $3.9 \AA$ for the hydrogen bonnding. But when we added the hydrogens to the protein at $\mathrm{pH} 7$, the hydrogen-acceptor distance criteria of $2.5 \AA$ was not met, due to which we were unable to see the hydrogen bonding in our model. 
Chain A

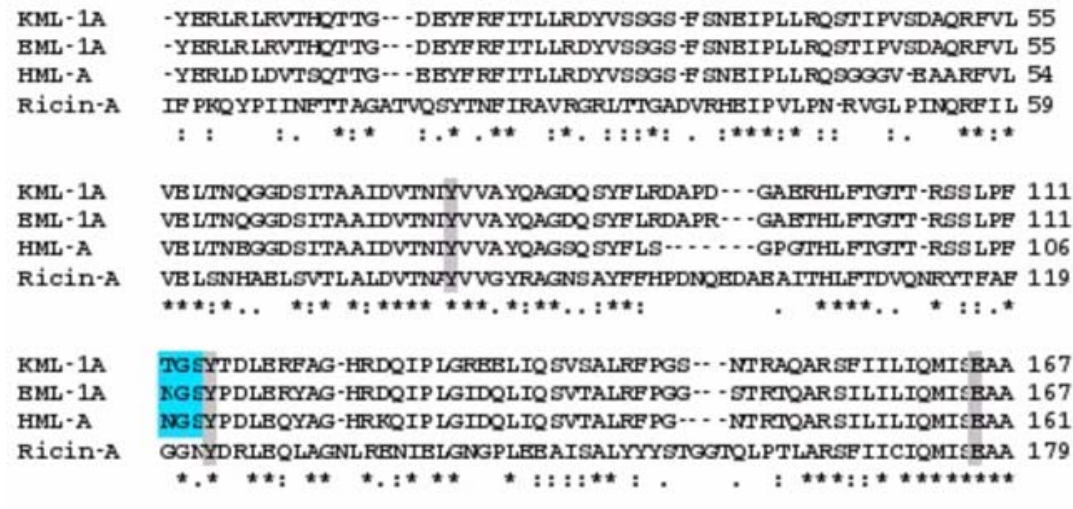

KML-1A RFNPILWRARQYI SSGGSFLPDTYILQLETSWGQQSTQVQHSTDGVFNNPIRUTISTGVF 227 BML-1A RFNPILWRARQYINSGASTLPDVYMLBLETSWOCQSTQVQHSTDGVFNNPIRLAIP PGNF 227 HML-A RFNPILWRARQYINSGASELPDVYMLBL ETSWGQQSTQVDQSTBGVENNPIRLAIP-GNE 220

Ricin-A FFQYIBGEMRTRIFCNRRSAPDPSVITLENSWGRLSTAIQBSNQGAFASPIQLQRFNGSK 239

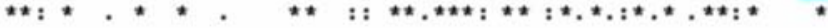

KML-1A VTLSNVRDVIASLATMLFVCEDRPSS- 254

BML-1A VTLINVRDVIASLATMLFVOG ….... 248

HML-A VTLINVRDVIASLATMLFVC........ 240

RICIN-A FSVYDVSILIPIIALMVYRCAPPPSSQE 267

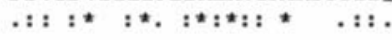

Chain B

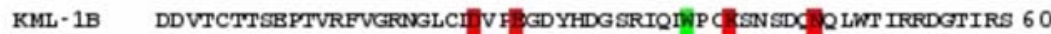
BML-1B DDVTC SASBPTVR IVGRAGMCU VF DD FRDGNQIQINP S SNND I 2 LWT IKRDGTIRS 60

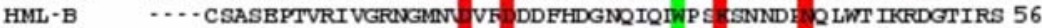

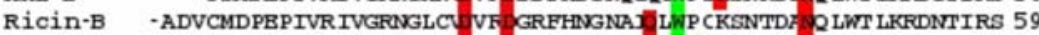

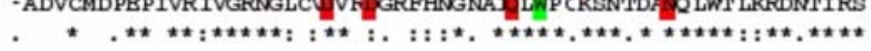

KML-1B NGRCL TYGYTAGS TMIYD CNRC EWD ITTWQ IRGNGIILNPRSMMVIGTPSGSRGTRGT 120

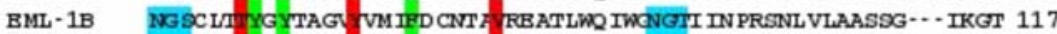
HML-B NGSCII YGYTAG VM IFD CNI F RBATIWQ IWONGII IN PRSNLALAASSG -.. IKGT 113

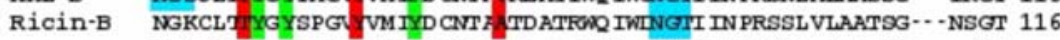

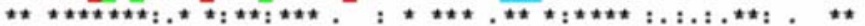

KML-1B TFTLQTLGYSLOQGWLANDTA PREVTIYGERDHCMETSGGKVWVGTCVSGKONOFWALY 180 BML-1B TLTVQTLDYTLOQGWLAANDTA PRBVTIYGEFDLCMBSNOGSVWVETCVSSOKNOFWALY 177 HML-B TLTVQTLDYTLOQGWLACNDTA PREVTTYGENDLCMBSNOGSVWVETCVS-QQNDFWALY 172 RICIN-B TLTVQTNTYAVSQGWLPINNIQ PEVTTIVG LYGLCLQANSOQVWIEDCSSEKABCOWALY 176

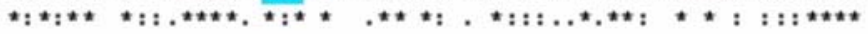

KML-1B GDGSIRPKPYQDQ CITSOGDSVRSVINLFSCTAGSPROFOVFTNKGAILNLKNRLAD DVA 240 BML-1B GDGSIRPKQNODQ CITOGEDSVIVINIVSCSAGSSGOFWVTNBGAILNLKANGLAL VA 237 HML-B GDGSIRPEQNQDQ CLTSGRDSVAG-INIVSCSGGSSGQFWVFT NBGAILNLKNGLAL-VA 231 RICIN-B ADGSIRPQQNRDNCLTSDSNIFETVVKI LSOGPASSGOFOMIKNDGTILNLYSGLVI VR 236

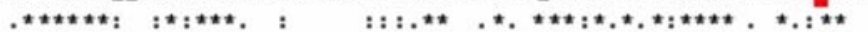

KML-1B BSNPSLRRIIIFSVTGNH QNMWL PVP 266

BML-1B ANPKLRRIITYPATGKH QMNL PVP 263

HML-B -NPGLGQIITYPATGKH QMNL PVP 255

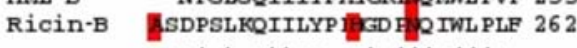

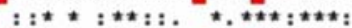

Fig. 5. Multiple sequence alignment of KML, EML-1, HML and Ricin. Key residues in the catalytic site are shadowed by grey color, residues that form hydrogen bonds with galactose are shadowed by red color, residues that provide the hydrophobic contact are shadowed by green color and residues involved in the glycosylation are shadowed by turquoise color.

\section{Discussion}

In recent years, due to its potential medical application in cancer therapy, studies concerning the biological effects of the mistletoe lectins have been drawing attention. The A-chain of the heterodimeric mistletoe lectins appears to exert apoptotic cell killing in cancer cells upon its entry to cells via potential receptor internalization through the involvement of the Bchain with sugar binding properties. The physiological function of the type II RIPs has been shown to inhibit protein synthesis, modulate telomerase, and regulate the phosphorylation of protein kinases in mammalian cells (Choi et al., 2004; 


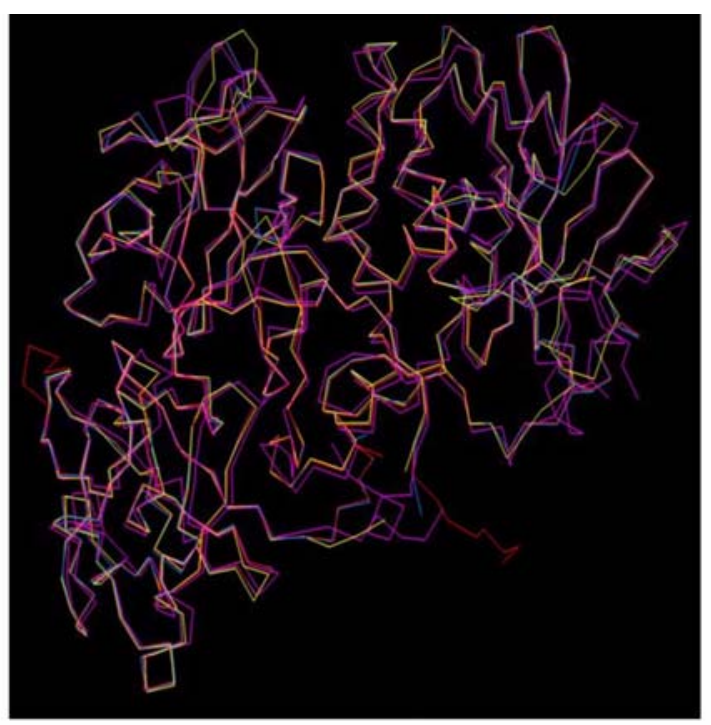

(A)

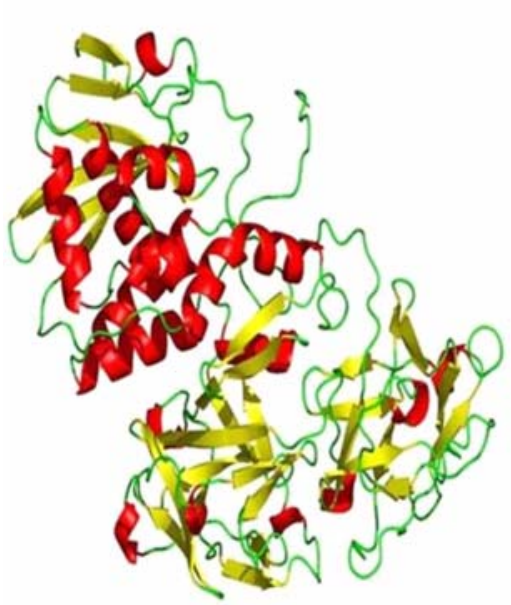

(B)

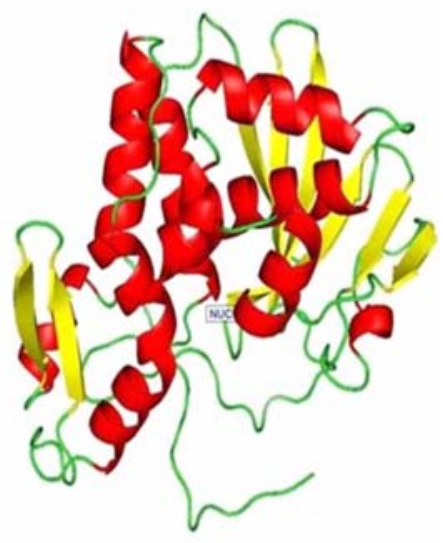

(C)

Fig. 6. 3D structure of KML by homology modeling. (A) Structural alignment of $\mathrm{C}^{\alpha}$ trace of KML, EML-1, HML and Ricin. KML, EML-1, HML and Ricin are represented by Red, Blue, Yellow and Pink color respectively. Fig. 6A was generated using InsightII version 2000.3L (InsightII, 2003). (B) 3D structural model of the KML is shown. (C) Rectangle indicates the nucleotide binding site ("NUC") for the KML-1A. Fig. B and C were generated using PyMol version 0.97 (Delano).

Narayanan et al., 2005). Yet, the detailed molecular basis for the biological effects of the mistletoe lectins is unclear. In this work, to better understand and define the function of the mistletoe lectins, we made efforts to study less well defined KML. Our results demonstrated that the rKML-1A (isoform-1 of KML-A-chain) was expressed in a soluble form, and the purified rKML-1A was stable. On the other hand, our attempts to purify rKML-1B (isoform-1 of KML-B-chain) have failed. Currently, efforts are being made to modify our system for the expression of the B-chain. Our CD and NMR data suggested that the purified A-chain showed characteristics of a properly folded protein and was ready for biochemical and structural studies. Using the purified rKML-1A, we first evaluated the ribosome-inactivating activity. Our data showed that the $\mathrm{IC}_{50}$ of rKML-1A is comparable to that of the native KML. Our cell proliferation assay indicates that the rKLM-1A is active in exerting cytotoxicity in cancer cells. However, the efficiency of the KML-A1 using the artificial delivery system appears to be lower compared to the native KML.

Type II RIPs are highly homologous among the family members (Park et al., 2001). This high degree of the conserved sequence led us to attempt homology modeling of KML. Based on the stereochemical quality and residue-byresidue geometry checked by PROCHECK, overall, the main chain fold of KML is well conserved as compared to the Xray structure of EML-1 (Niwa et al., 2003). The alignment of the amino acid sequences indicates that the key catalytic residues of the A-chain of EML-1 are well conserved in the 

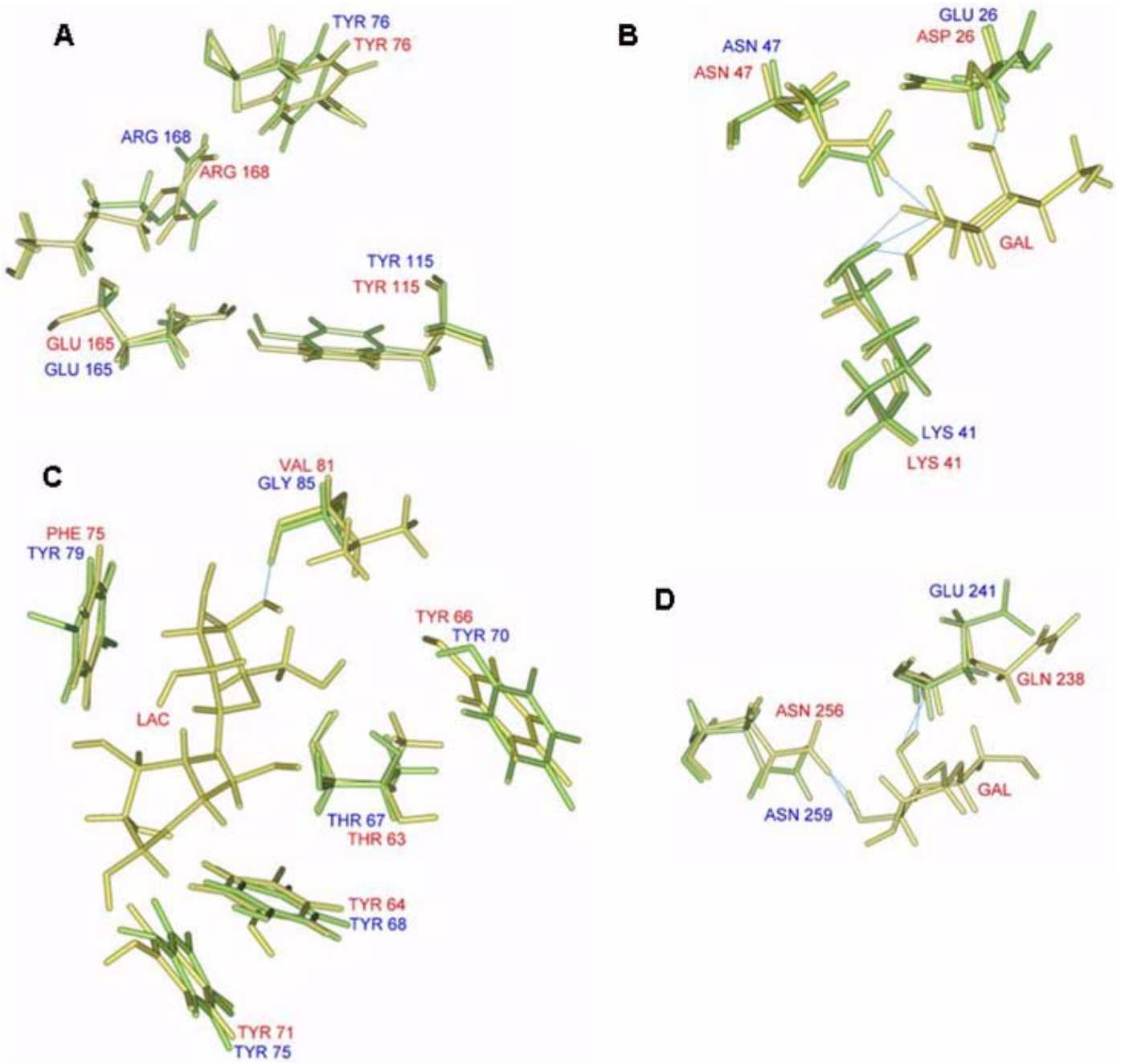

Fig. 7. Analysis of the active site of KML-1A and sugar-binding sites of KML-1B. (A) The active site residues of the KML-1A (green) superimposed with the active site residues of the A-chain of EML-1 (yellow). Annotations for the EML-1A and KML-1A are indicated by red and blue color, respectively. (B) Superimposition of N-terminal galactose binding sites of KML-1B. Superimposition of Nterminal galactose binding site residues of KML-1B (green) with EML-1B (yellow). Galactose (GAL) is shown in yellow color and the corresponding hydrogen bonding between galactose and EML-1B is indicated in dashed lines. Annotations for the EML-1B and KML$1 \mathrm{~B}$ are indicated by red and blue color, respectively. (C) Superimposition of Lactose binding residues of KML-1B (green) with HML-B (yellow). Lactose (LAC) is shown in yellow color and the corresponding hydrogen bonding between galactose moeity of lactose and HML-B is indicated in dashed lines. Annotations for the HML-B and KML-1B are indicated by red and blue color, respectively. (D) Superimposition of C-terminal galactose binding site residues of KML-1B (green) EML-1B (yellow). Galactose (GAL) is given yellow color and the corresponding hydrogen bonding between galactose and EML-1B is indicated in dashed lines. Annotations for the EML$1 \mathrm{~B}$ and KML-1B are indicated by red and blue color, respectively. Figures were generated using InsightII version 2000.3L (InsightII, 2003).

active site of KML-1A as well as other RIPs (Katzin et al., 1991). Our structural model confirms the conservation of catalytic sites in both EML-1 and KML (Fig. 7A). The Asn112 of the A-chain of EML-1 was shown to be engaged in the N-linked glycosylation (Niwa et al., 2003). It is noted, on the other hand, that the Asn112 is changed to Thr112 in KML-A1 instead (Fig. 5). Whether the mutation in the glycosylation site on the A-chain of the lectins has any influence on the overall catalytic activity of KMLA remains to be answered.
The B-chains of the type II RIPs were shown to contain few sugar binding sites. They share a high degree of sequence similarity, and so are their 3-dimensional structures. However, their sugar binding specificities appear to vary. These differences in the specificities might be important (Frankel et al., 1996; Steeves et al., 1999), because the biological effects of the type II RIPs appear to be determined by the binding of the Bchains to the potential sugar-containing receptors on the cell surface. The structural superimposition of the B-chain of EML-1 with KML-1B reveals that some differences at the 
both $\mathrm{C}$ - and N-terminal sites exist. At the N-terminal site while Lys41, Asn47 and Trp38 are conserved, Asp26 is changed to Glu26 in KML-1B instead. At the C-terminal site while Asn256 is conserved, Gln238 is changed to Glu241 in KML-1B (Fig. 7B, D). Since only the main chain atoms of Asp26 and Gln238 in the B-chain of EML-1 are involved in the hydrogen bonding, the above mentioned mutations are expected to affect little on the molecular interaction of the Bchains with the sugar. Also from our studies at the third sugar binding pocket recently identified in HML-B, all the residues at the third sugar binding site are absolutely conserved in EML-1B while the residues mutated in KML-1B are Gly85, Tyr79. Since only main chain amide nitrogen of Val81 in HML-B is involved in the hydrogen bonding, the corresponding residue mutated into Gly85 in KML-1B is speculated to make little influence on the binding of lactose. Also the Phe75 in HML-B mutated to Tyr79 in KML-1B can still provide the hydrophobic contact for galactose moeity of lactose. Taken together, our studies suggest that the galactose binding sites of KML-1B are similar but not the same compared to that of EML1. Apart from sugar-binding specificities, we have noticed that the B-chains of EML-1, HML contains three N-linked glycosylation sites, Ricin has two N-linked glycosylation sites while KML-B1 is predicted to contain only one N-glycosylation site (Fig. 5) and whether the absence of the two Nglycosylation sites in the KML-1B has any influence on its biological function might be worthy of further investigation. A future study involving the sugar binding characteristics of B-chain of KML might provide a clue for the better comprehension on the biological effects of KML and its molecular basis in inducing apoptotic cell death in cancer cells.

Acknowledgment This work is partly supported by grant (R05-2002-000-00662-0) to S.K. Song from the Basic Research Program of the Korea Science \& Engineering Foundation.

\section{References}

Choi, S. H., Lyu, S. Y. and Park, W. B. (2004) Mistletoe lectin induces apoptosis and telomerase inhibition in human A253 cancer cells through dephosphorylation of Akt. Arch. Pham. Res. 27, 68-76.

DeLano W. L. "The PyMOL Molecular Graphics System." DeLano Scientific LLC, San Carlos, CA, USA. http://www. pymol.org.

Eck, J., Langer, M., Mockel, B., Baur, A., Rothe, M., Zinke, H. and Lentzen, H. (1999) Cloning of the mistletoe lectin gene and characterization of the recombinant A-chain. Eur. J. Biochem. 264, 775-784.

Eck, J., Langer, M., Mockel, B., Witthohn, K., Zinke, H. and Lentzen, H. (1999) Characterization of the recombinant and plant-derived mistletoe lectin and their B-chains. Eur. J. Biochem. 265, 788-797.
Endo, Y., Tsurugi, K. and Franz, H. (1988) The site of action of the A-chain of mistletoe lectin I on eukaryotic ribosomes. The RNA N-glycosidase activity of the protein. FEBS Lett. 231, 378-380.

Frankel, A. E., Burbage, C., Fu, T., Tagge, E., Chandler, J. and Willingham, M. C. (1996) Ricin toxin contains at least three galactose-binding sites located in B chain subdomains 1 , 1 , and 2. Biochemistry 35, 14749-14756.

Hajto, T., Hostanska, K., Frei, K., Rordorf, C. and Gabius, H. J. (1990) Increased secretion of tumor necrosis factors alpha, interleukin 1, and interleukin 6 by human mononuclear cells exposed to beta-galactoside-specific lectin from clinically applied mistletoe extract. Cancer Res. 11, 3322-3326.

InsightII, version 2000.3L. San Diego: Accelrys Inc. 2003.

Katzin, B. J., Collins, E. J. and Robertus, J. D. (1991) Structure of

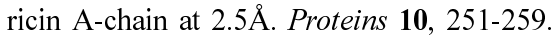

Krauspenhaar, R., Eschenburg, S., Perbandt, M., Kornilov, V., Konareva, N., Mikailova, I., Stoeva, S., Wacker, R., Maier, T., Singh, T., Mikhailov, A., Voelter, W. and Betzel, C. (1999) Crystal Structure of Mistletoe Lectin I from Viscum album. Biochem. Biophys. Res. Commun. 257, 418-424.

Langer, M., Rothe, M., Eck, J., MÖckel, B. and Zinke, H. (1996) A Nonradioactive Assay for Ribosome-Inactivating Proteins. Anal. Biochem. 243, 150-153.

Laskowski, R. A., MacArthur, M. W., Moss, D. S. and Thornton, J. M. (1993) PROCHECK: a program to check the stereochemical quality of protein structures. J. Appl. Cryst. 26, 283-291.

Lyu, S. Y., Choi, S. H. and Park, W. B. (2002) Korean mistletoe lectin-induced apoptosis in hepatocarcinoma cells is associated with inhibition of telomerase via mitochondrial controlled pathway independent of p53. Arch. Pham. Res. 25, 1-8.

Lyu, S. Y., Park, W. B., Choi, K. H. and Kim, W. H. (2001) Involvement of caspase- 3 in apoptosis induced by Viscum album var. Coloratum agglutinin in HL-60 cells. Biosci. Biotechnol. Biochem. 65, 543-541.

Mishra, V., Bilgrami, S., Sharma, R. S., Kaur, P., Yadav, S., Krauspenhaar, R., Betzel, C., Voelter, W., Babu C. R. and Singh, T. P. (2005) Crystal Structure of Himalayan Mistletoe Ribosome-inactivating Protein Reveals the Presence of a Natural Inhibitor and a New Functionally Active Sugar-binding Site. J. Biol. Chem. 280, 20712-20721.

Montfort, W., Villafranca, J. E., Monzingo, A. F., Ernst, S. R., Katzin, B., Rutenber, E., Xuong, N. H., Hamlin, R. and Robertus, J. D. (1987) The three-dimensional structure of ricin

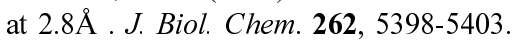

Narayanan, S., Surendranath, K., Bora, N., Surolia, A. and Karande, A. A. (2005) Ribosome inactivating proteins and apoptosis. FEBS Lett. 579, 1324-1331.

Niwa, H., Tonevitsky, A. G., Agapov, I. I., Saward, S., Pfuller, U. and Palmer, R. A. (2003) Crystal structure at $3 \AA$ of mistletoe lectin I, a dimeric type-II ribosome-inactivating protein, complexed with galactose. Eur. J. Biochem. 270, 2739-2749.

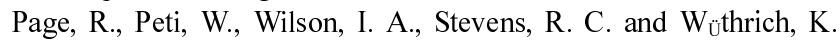
(2005) NMR screening and crystal quality of bacterially expressed prokaryotic and eukaryotic proteins in a structural genomics pipeline. Proc. Natl. Acad. Sci. USA 102, 1901-1905.

Park, C. H., Lee, D. W., Kang, T. B., Lee, K. H., Yoon, T. J., Kim, J. B., Do, M. S. and Song, S. K. (2001) cDNA cloning and sequence analysis of the lectin genes of the Korean 
mistletoe (Viscum album coloratum). Mol. Cells. 12, 215-220.

Peumans, W. J., Hao, Q. and Van Damme, E. J. M. (2001) ribosome-inactivating proteins from plants: more than RNA Nglycosidases? FASEB J. 15, 1493-1506.

Sali, A. and Blundell, T. L. (1993) Comparative protein modeling by satisfaction of spatial restraints. $J$. Mol. Biol. 234, 779-815.

Schumacher, U., Feldhaus, S. and Mengs, U. (2000) Recombinant mistletoe lectin (rML) is successful in treating human ovarian cancer cells transplanted into severe combined immunodeficient (SCID) mice. Cancer Lett. 150, 171-175.

Steeves, R. M., Denton, M. E., Barnard, F. C., Henry, A. and Lambert, J. M. (1999) Identification of three oligosaccharide binding sites in ricin. Biochemistry 38, 11677-11685.

Thompson, J. D., Higgins, D. G. and Gibson, T. J. (1994)
CLUSTAL W: improving the sensitivity of progressive multiple sequence alignment through sequence weighting, position-specific gap penalities and weight matrix choice. Nucleic Acids Res. 22, 4673-4680.

Yoon, T. J., Yoo, Y. C., Choi, O. B., Do, M. S., Kang, T. B., Lee, S. W., Azuma, I. and Kim, J. B. (1995) Inhibitory effect of Korean mistletoe (Viscum album coloratum) extract on tumour angiogenesis and metastasis of haematogenous and nonhaematogenous tumour cells in mice. Cancer Lett. 97, 83-91.

Yoon, T. J., Yoo, Y. C., Kang, T. B., Shimazki, K., Song, S. K., Lee, K. H., Kim, S. H., Park, C. H., Azuma, I. and Kim, J. B. (1999) Lectins isolated from Korean mistletoe (Viscum album coloratum) induce apoptosis in tumor cells. Cancer Lett. 136, 33-40. 